\title{
ARTICLE
}

\section{Exploring the origins of crystallisation kinetics in hierarchical materials using in situ X-ray diffraction and Pair distribution function analysis}

Received 00th January 20xx, Accepted 00th January 20xx DOI: $10.1039 / x 0 x \times 00000 x$
Matthew E. Potter, ${ }^{a}$ Mark E. Light, ${ }^{* a}$ Daniel J. M. Irving, ${ }^{a}$ Alice E. Oakley, ${ }^{a}$ Stephanie Chapman, ${ }^{\text {a }}$ Philip Chater, ${ }^{\mathrm{b}}$ Geoff Cutts, ${ }^{\mathrm{b}}$ Andrew Watts, ${ }^{\mathrm{b}}$ Michael Wharmby, ${ }^{\mathrm{b}}$ Bart D. Vandegehuchte, ${ }^{\mathrm{c}}$ Moritz Schreiber ${ }^{\mathrm{c}}$ and Robert Raja ${ }^{\mathrm{a}}$

\begin{abstract}
The discovery of novel catalytic materials is predicated on understanding contemporary synthetic processes. With this fundamental knowledge in place it becomes possible to modify the final material with subtle changes to the synthesis process. In this vein hierarchical materials, formed by the addition of a mesoporogen within the hydrothermal synthesis have attracted a significant amount of attention due to their catalytic benefits over analogous microporous species. In this work we monitor the hydrothermal synthesis in situ of a hierarchical and a microporous aluminophosphate, for the first time, combining total scattering and pairwise distribution function data. In doing so we observe the local formation of the species, and the longer range crystallisation processes concurrently.
\end{abstract}

\section{Introduction}

Understanding the behaviour of catalytic materials is vital in tailoring their properties for improved activity. A wide range of both characterisation and computational studies have been dedicated to understanding active-site behaviour, reactant binding and the formation of intermediates. ${ }^{1,2}$ The evolution of characterisation techniques facilitates improved resolution on the local structure environment, allowing a better understanding of both the materials, and the catalytic pathways they promote. $^{3}$ The continued improvements in characterisation resolution has also developed alongside a wider range of sample environments, allowing realistic reaction conditions to be realised. These technological developments have overseen a massive boom in both in situ and, more recently, operando characterisation studies, as higher temperatures, pressures and environments have become possible. ${ }^{4,5,6,7}$

While the catalytic behaviour of materials is widely studied, the underlying reasons of how variations in synthetic parameters can influence framework formation and catalytic activity are relatively unknown..$^{8,9}$ To this day even the synthesis of many industrially used catalysts is still regarded as a 'black box'. This is particularly true in the case of microporous materials such as zeolite (aluminosilicates)

a. University of Southampton, Chemistry Department, Southampton, Hants, SO17 1BJ, UK. E-mail: light@soton.ac.uk

b. Diamond Light Source, Harwell Science and Innovation Campus, Didcot, Oxon OX11 ODE, UK

Total Research \& Technology Feluy, Zone Industrielle Feluy C, B-7181 Seneffe, Belgium

†Electronic Supplementary Information (ESI) available: See

DOI: $10.1039 / x 0 x x 00000 x$ and analogous aluminophosphate (AIPO) frameworks. Many studies have investigated the link between catalytic activity and synthesis parameters such as $\mathrm{pH}$, reagent choice and temperature, for these materials. ${ }^{10,11,12}$ While these provide significant insights for both catalyst and active-site design, this ex situ approach yields limited information on the design of porous materials. The concept of zeolites and AIPOs synthesis is quite simple. Inorganic $\mathrm{Si}, \mathrm{Al}$ or $\mathrm{P}$ reagents form a gel containing oxidic tetrahedral primary building units (PBUs). These PBUs combine, sharing an oxygen, to form either $\mathrm{Si}-\mathrm{O}-\mathrm{Si}$, Si-O-Al or, in the case of AlPOs, Al-O-P bridges. These bridges then assemble into simple rings or shapes called secondary building units (SBUs), which then arrange into a specific framework topology. The precise framework is dictated by the exact synthesis conditions and by a templating agent, which the framework forms around. The result is a microporous species with tetrahedral framework atoms ( $P$, $\mathrm{Al}$ or $\mathrm{Si}$ ) connected by oxygen bridges. Despite this, several key questions remain about the exact synthesis pathways. Understanding how to preferentially form one SBU over another, or how to combine SBUs in a certain configuration are still major challenges. ${ }^{13}$

The difficulty in understanding AIPO and zeolite synthesis is that hydrothermal synthesis has not previously translated well to in situ set-ups, requiring high temperatures of $150-200{ }^{\circ} \mathrm{C} .{ }^{14,15}$ While these temperatures are achievable with modern sample environments, the associated pressure build-up within the confined system is a significant challenge, as pressures can reach 15 bar from steam formation alone. Further, the diluted synthesis gel makes it harder to focus on the active sites that are of interest. The framework atoms themselves are also quite 'light', therefore, will not easily stand out from the rest of the gel. Despite this a range of characterisation 
techniques have created elegant configurations to overcome these challenges. Solid state NMR (SSNMR) has been used in probing the formation mechanism of AIPO and zeolite species in situ, since the development of high-temperature sealed rotors. ${ }^{16,17}$ Particularly ${ }^{31} \mathrm{P}$ ssNMR work has shown the changing distribution of $\mathrm{P}$ environments from $\mathrm{PO}_{4}$ to $\mathrm{P}(\mathrm{OAl})_{4} .{ }^{17}$ While ssNMR focuses on the atomic scale changes that occur during crystallisation a range of microscopy techniques are rapidly evolving to a point where in situ hydrothermal synthesis can be observed. ${ }^{18}$ To the point of framework growth, one SBU at a time has been observed. ${ }^{19}$

Synchrotron-based characterisation commonly provides the high signal to noise ratios required for monitoring the in situ crystallisation of these materials, while the versatile nature of many beamlines can also accommodate an increasing range of sample environments. The popular techniques for such studies include $\mathrm{X}$-ray absorption spectroscopy (XAS), X-ray diffraction (XRD) and small and wide-angle $X$-ray scattering (SAXS and WAXS). ${ }^{20,21,22}$ XAS studies have been primarily focused on the formation of metal doped AIPOs, as the dopant (e.g. Co, Ru or $\mathrm{Zn}$ ) can be readily distinguished from the lighter atoms in the gel. ${ }^{20,23,24}$ This has allowed groups to observe the progression from the metal salt precursor, to tetrahedral framework species, coinciding with the framework formation. AI XAS studies have also been reported; however these were limited to XANES analysis, due to the lower energy required. ${ }^{25} \mathrm{X}$-ray scattering experiments (SAXS and WAXS) have been used to focus more on the development of crystal aggregates from PBUs, where kinetic information of crystal growth on crystal surfaces has been vital in revealing the rate determining step. ${ }^{22}$

XRD is a powerful technique for observing the evolution of a crystalline framework. As the observed Bragg peaks correspond to crystalline phases, thus one can focus purely on emerging crystalline products, and not the amorphous synthesis gel. Studies of this nature have been ongoing for the last few decades, as XRD has few restrictions on sample environment. ${ }^{26}$ Early studies on AlPO formation were able to determine crystallisation kinetics, and crystallisation activation energies from the peak intensities. ${ }^{27}$ This revealed that dopant choice ( $\mathrm{Co}$ or $\mathrm{Mn}$ ) heavily influences the activation energy, while the addition of HF drastically decreases the activation energy. ${ }^{27}$ However, another reason for the interest in XRD, and other X-ray-based techniques is the ability to combine them for simultaneous data acquisition. ${ }^{20,24,26,27}$ Perhaps most common is the coupling of XAS and XRD, though other combinations have also been achieved. ${ }^{28}$ Ultimately the simultaneously use of techniques provides information over a wide length scale, focussing simultaneously on the crystal growth, and the atomistic pathway followed. Such studies have been invaluable at identifying different regimes of the crystalline process, most notably in ZnAIPO-34 and CoAIPO-5.20,24,29

A technique commonly coupled with XRD is pair distribution function (PDF), written as $D(r),{ }^{30}$ and total scattering. These provide information on the atomic distances within a material, with $D(r)$ representing the probability of finding an atom at a specific distance, $r$, from the centre of another atom.

$$
D(r)=4 \pi r\left(\rho(r)-\rho_{0}\right)
$$

Where $r$ is the atomic distance, $\rho(r)$ is the microscopic pair densities, $\rho_{0}$ is the average atomic pair density of the material. Using $D(r)$ one can extract both the distribution and density of atoms, and therefore the local structure of a material. This can be used in conjunction with $X R D$, giving an insight on the long-range crystalline structure, allowing for the full structural analysis of ordered, disordered and amorphous solids, solutions and liquids. ${ }^{31,32}$

PDF has been used ex situ to explore a wide range of zeotype materials, providing in-depth structural information on a wide range of systems, including establishing the true form of zeolite beta. ${ }^{33}$ PDF analysis has been used to great effect to explore the structure of more recent zeolites, or germanosilicate zeolite precursors, undergoing the contemporary ADOR (assembly-disassemblyorganisation-reassembly) technique. ${ }^{34,35,36}$ In comparison there are few in situ PDF studies, owing to the need to contain the sample in a thin-walled, sealed, capillary. Recent work by Wakihara et al. demonstrated the ability to incorporate a microwave reactor with the BL08W beamline at SPring-8 to form both beta and MFI zeolites. ${ }^{37}$ In this study they were able to use the PDF to resolve the different SBUs formed in two different reactions. To our knowledge there are currently no reports of a non-microwave heated capillary for monitoring the in situ synthesis of zeotype species. However, such information would be invaluable in understanding hydrothermal synthesis processes.

Understanding the synthesis protocols of traditional or model systems, will provide vital information on common themes and processes in material synthesis. This is particularly pertinent due to the increasing numbers of novel species, where subtle synthetic changes can have significant impacts on catalytic behaviour. One area where this is highly relevant is the development of hierarchically porous materials. Hierarchical materials are typically based on microporous materials, such as AIPOs or zeolites, which also accommodate mesopores within the framework. ${ }^{38,39} \mathrm{~A}$ wide range of methods have been explored to create these systems, including postsynthetic top-down methods, to introduce mesopores into zeotype species. ${ }^{40}$ In contrast bottom-up approaches require fewer synthetic steps, as the mesopores form around surfactants, or hard templates (mesoporogens) during the synthesis procedure. Thus, adding comparatively small quantities of mesoporogen, can alter the physicochemical properties of the catalyst by modifying the pore volume or surface texture. These modifications have facilitated catalytic improvements including extended catalytic lifetimes, modifying selectivity and introducing distinct acid sites within the mesopores. ${ }^{41,42,43}$ Despite growing interest in hierarchical materials, little is known about their synthesis, particularly the role of added mesoporogens, and how this differs from the synthesis of standard microporous AIPOs. Therefore, in this work we compare and contrast the synthesis mechanisms of hierarchical AlPO-5 (HP-AlPO-5) and traditional microporous AIPO-5 (MP-AIPO-5). To do so we have combined in situ XRD (specifically, total scattering) and PDF to understand both the short- and long-range order of the systems. We 
will discuss the influence of the mesoporogen, DMOD (dimethyloctadecyl[3-(trimethoxysilane)propyl]ammonium chloride, on the synthesis pathways and also the design of our experimental setup, allowing us to monitor the hydrothermal synthesis in situ at $200{ }^{\circ} \mathrm{C}$.

\section{Experimental}

\section{In situ PDF experiments to track crystallisation}

$0.9 \mathrm{~mL}$ of $\mathrm{H}_{3} \mathrm{PO}_{4}$ (85 wt. \% in water) was diluted in $4 \mathrm{~mL}$ of deionised water and stirred for 5 minutes. $2.7 \mathrm{~g}$ of ground aluminium isopropoxide was added gradually to the diluted acid and left to stir for 1 hour. $1.5 \mathrm{~mL}$ of triethylamine was added to the gel, followed by a further $8 \mathrm{~mL}$ of deionised water. In the case of the hierarchically porous (HP) AlPO-5 gel, $0.86 \mathrm{~mL}$ of dimethyloctadecyl[3(trimethoxysilyl)propyl]ammonium chloride solution (42 wt. \% in methanol) was added before the triethylamine. The gel was left to stir overnight.

The gel was loaded into the hydrothermal cell on the I15-1 beamline at Diamond Light Source (Figure S1), consisting of a fused quartz tube mounted in an aluminium heating block. The sample was contained within the fused quartz tube $(2.5 \mathrm{~mm}$ diameter, $0.1 \mathrm{~mm}$ wall thickness, $50 \mathrm{~mm}$ long, Friedrich \& Dimmock inc.) which acts as the pressure vessel under hydrothermal conditions. The gel was loaded so that only $\sim 0.5 \mathrm{~cm}$ of the tube was filled in order to minimise pressure build up during the crystallisation. The tube was sealed into an aluminium stopper using custom made Teflon ferrules and an M6 Derlin nut (IDEX). The sealed tube was then mounted into an aluminium block which was heated using two $100 \mathrm{~W}$ cartridge heaters (RS components). The aluminium block housing the tube has additional aluminium inserts to ensure that the tube remains straight and to provide good thermal contact over a large area of the tube; this allows for even heating of the sample tube. A thermocouple can then be attached to the capillary and the whole arrangement held in place with an aluminium lid. A slit on the upstream face of the heated block provides the entrance window for the incident X-rays. A square hole on the aluminium lid is designed to perfectly match the detector geometry used on I15-1 and allows the scattered X-rays to pass unimpeded from the sample tube. The result is that there is no impact on the scattered radiation from the aluminium housing, even at the very high scattering angles required for quality PDF data collection (up to a $2 \theta$ of $50^{\circ}$ using the geometry on the $115-1$ beamline, equating to a maximum $Q$ of $32.8 \AA^{-1}$ at the typical working energy of $76.69 \mathrm{keV}$ ), and the data is of equal quality to a sample collected in the same tube in absence of the aluminium block.

The temperature is controlled using a custom-built power supply fitted with Eurotherm 3504 controllers. The system temperature is typically controlled with a K-type thermocouple fixed to the aluminium housing block, and the sample temperature is monitored with a second thermocouple attached to the sample tube. The tip of the sample thermocouple is in direct contact with the outside edge of the sample tube, providing the internal sample temperature to within a few degrees (see Figure S1). If more precise temperature control is required, the sample temperature inside the tube may be calibrated using a standard with a known thermal expansion or phase transition. PID control allows for heating and cooling rates to be applied with fair precision.

The hydrothermal cell containing the gel sample was heated from 35 ${ }^{\circ} \mathrm{C}$ to $205^{\circ} \mathrm{C}$ at a ramp rate of $2{ }^{\circ} \mathrm{C} \mathrm{min}-1$ and held at $205{ }^{\circ} \mathrm{C}$; a small temperature gradient between the aluminium block and the sample tube gave the desired sample synthesis temperature of $200{ }^{\circ} \mathrm{C}$. This procedure was then repeated with each component of the synthesis gel in isolation, with the solid aluminium isopropoxide being dissolved in a minimum amount of deionised water.

This synthesis was chosen to mimic our ex situ synthesis of these materials, which is known to yield a phase-pure AIPO-5 framework (Figure S2). In the case of HP AlPO-5 $\mathrm{N}_{2}$ physisorption shows clear evidence of both microporosity and mesoporosity, whereas the MP AIPO-5 system is exclusively microporous (Figures S3 and S4).

The hydrothermal cell was mounted onto the I15-1 sample stage and aligned with the capillary mounted vertically so that the X-ray beam passed through the gel sample. I15-1 operates with two area detectors, one placed near to the sample for PDF analysis and one further from the sample for higher resolution Bragg data. The near detector (Perkin Elmer, XRD4343CT) was placed $200 \mathrm{~mm}$ from the sample in a diamond orientation, so that the beam was centred on the lower corner of the diamond. The far detector (Perkin Elmer XRD 1611 CP3) was placed $850 \mathrm{~mm}$ from the sample so that the upper edge of the detector was centred on the beam. X-ray total scattering data were collected using both detectors simultaneously at an energy of $76.69 \mathrm{keV}(\lambda=0.161669 \AA)$. Each individual data collection during the temperature ramp and hold was $60 \mathrm{~s}$. The area detector data was automatically corrected for dark current, flat field, beam polarisation and transmission before integrating into a 1D dataset using routines within the DAWN software. ${ }^{44}$ The $1 \mathrm{D}$ data was further processed using the GudrunX software ${ }^{45}$ over the range $0.8 \leq \mathrm{Q} \leq$ $22.5 \AA^{-1}$ to correct for background, multiple scattering, Compton scattering and absorption before Fourier transform to produce the PDF dataset, $D(r) .45$

\section{Results and discussion}

\section{Total scattering data}

The pre-prepared synthesis gels for both microporous AIPO-5 (MP AIPO-5) and hierarchical AIPO-5 (HP AIPO-5) were individually loaded into the I15-1 hydrothermal cell (see ESI for further details). The Xray data collection started when the material was at room temperature (0 minutes). The systems were then held at room temperature for 10 minutes, before heating at a rate of $2{ }^{\circ} \mathrm{C} \mathrm{min}^{-1}$ up to $200{ }^{\circ} \mathrm{C}$. On reaching $200{ }^{\circ} \mathrm{C}$ (roughly 100 minutes), the temperature was held for another 900 minutes to observe changes in the X-ray diffraction and PDF data with time. 


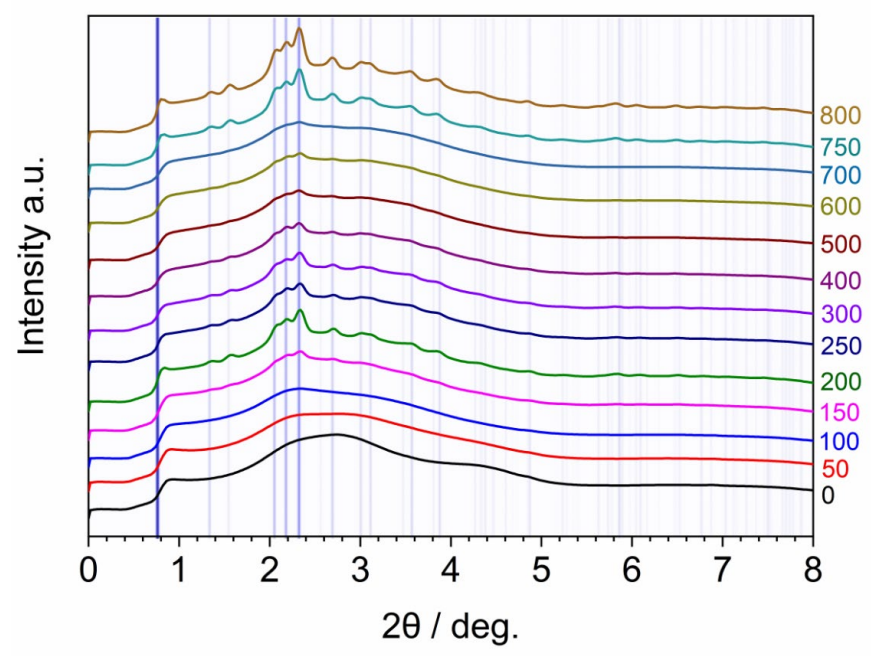

Figure 1: Stacked in situ diffraction data $(\lambda=0.161669 \AA$ - arbitrary $y$ offset), showing the evolution of Bragg peaks for the MP AIPO- 5 system as a function of time (minutes). Profiles superimposed on a calculated pseudo-film pattern for crystalline AIPO-5. ${ }^{46}$

Initially focussing on the X-ray diffraction data of MP AIPO-5, at room temperature ( 0 minutes) we see a relatively intense background, with no observable Bragg peaks, though we see some broad features at a $2 \theta$ of $\sim 2.5$ and $\sim 4.5^{\circ}$. This is the result of significant scattering from the liquid precursors, primarily the comparatively large quantities of water in the system. This additional information from the total scattering will be interrogated further in the form of its Fourier transform, the PDF, $D(r)$. The persistent feature at a $2 \theta$ of $\sim 0.85^{\circ}$ is where the beam-stop cuts into the scattered radiation and is therefore not a feature from the sample. As the time increases, we observe the emergence of Bragg peaks, due to the formation of crystalline material. Initially the most notable signal to appear is the triplet between a $2 \theta$ of $2-2.6^{\circ}$, corresponding to the (210), (002) and a combination of the (102), (211) and (300) hkl values respectively. This signal begins to appear as early as 140 minutes, which corresponds to having been at $200{ }^{\circ} \mathrm{C}$ for 40 minutes. However, it is possible to observe the beginnings of crystallisation at even earlier times, during the heating regime (50-100 minutes, 100$200{ }^{\circ} \mathrm{C}$ ) as the broad amorphous feature centred around $2.25^{\circ} 2 \theta$ begins to appear. It is noted that after 200 minutes, the intensity of the Bragg peaks begins to oscillate. We do not believe this is a genuine feature suggesting cycles of framework erosion and reconstruction. Instead this is likely due to the formation of gaseous bubbles within the capillary, which influences the quantity of sample in the beam, and thus the intensity of the corresponding signal. Indeed, these bubbles were observed during the experiments, suggesting while the current cell was highly effective, improvements could still be made to mitigate against bubbles and bumping. We note that maximum crystallinity occurs after 800 minutes, at which point a wide range of Bragg peaks are clearly visible up to a $2 \theta$ of $8^{\circ}$, exclusively corresponding to the AlPO-5 (AFI) framework. We emphasise that this is the most crystalline pattern we observed, however oscillations due to gaseous expansions stop us from drawing similar conclusions beyond 800 minutes. This confirms that the solid material in our system is phase pure and we have successfully formed just the intended AIPO- 5 material. The relative intensity of the Bragg peaks differs from those predicted by simulations, and what has previously been reported. ${ }^{47,48}$ Commonly the $(100)$ peak $\left(0.8^{\circ}\right)$ is the most intense signal, however in our data this is clearly far less intense that the triplet at $2 \theta$ of $2-2.6^{\circ}$. The relative peak intensities of powdered AIPO-5 samples are known to change on calcination, when comparing the 'as synthesised' with 'calcined' versions. ${ }^{49}$ This is attributed to the removal of the templating (structure directing) agent, resulting in the removal of electron density from the pores, enhancing the contrast between empty pores and framework (Figure S5). We believe a similar principle occurs here, as the intensity of the (100) peak is heavily influenced by the contents of the channels. This is readily seen in powders as the (100) peak is significantly more intense (doubling the height) once the template is removed (Figure S6). Small deviations in unit cell dimensions were also observed $\left(\Delta V=14.88(31) \AA^{3}\right)$, which are attributed to the relaxation of the unit cell on removal of the templating agent, and are consistent with a subtle change in symmetry, typical of the AlPO- 5 framework. ${ }^{50}$ Thus, in aqueous synthesis conditions these channels will be filled with both template and water, therefore greatly lowering the intensity of this peak.

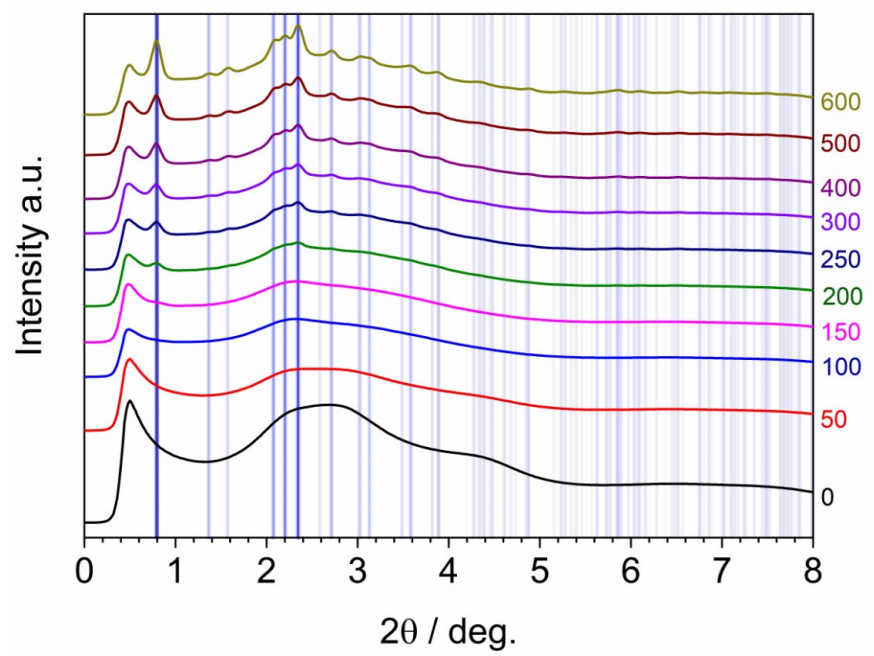

Figure 2: Stacked in situ diffraction data $(\lambda=0.161669 \AA$ - arbitrary y offset), showing the evolution of Bragg peaks for the HP AIPO-5 system as a function of time (minutes). Initial peak is an artefact caused by the beam-stop. Profiles superimposed on a calculated pseudo-film pattern for crystalline AIPO-5. ${ }^{46}$ 
As with the MP AIPO-5 system (Figure 1), the HP AIPO-5 system still has a large a background (Figure 2), which is visible from the 0 minutes sample (room temperature). Similar to the MP AIPO-5 system, the earliest indicator of emerging crystallinity for the HP AIPO- 5 is the appearance of the broad feature at around $2 \theta$ of 2.25 ${ }^{\circ}$, which again subsequently develops into the triplet of peaks at $2 \theta$ of 2-2.6 $6^{\circ}$. This is also accompanied by a developing signal at $2 \theta \sim 0.8$ ${ }^{\circ}$, which forms the (100) peak in the AIPO-5 pattern. We note in the HP AIPO-5 system the (100) peak appears more intense than the MP AIPO-5 system. However direct comparison of the two species shows that this may be due to weaker overall scattering in the HP system, caused by a the amorphous mesopores lowering the crystallinity of the system (Figure 3). Following the evolution of the Bragg peaks as a function of time, any semblance of crystallinity can only be seen after 150 minutes $\left(50\right.$ minutes at $200^{\circ} \mathrm{C}$ ). Further this only translates into obvious Bragg peaks after 200 minutes ( 100 minutes at $200^{\circ} \mathrm{C}$ ), suggesting that the HP AIPO-5 system forms at a slower rate than the MP AlPO-5 system. This finding is particularly interesting as the only difference between the synthesis gels is the inclusion of a small quantity of DMOD in the HP AIPO-5 system. However, this small change can noticeably retard the crystallisation kinetics of the HP AIPO-5 system, compared to the MP AIPO-5. Unlike the MP AIPO-5 system, the increased intensity of the HP AIPO- 5 Bragg peaks follows a more linear progression. However, the data collection was hindered after this time due to bubble formation, lowering the overall scattering data for the system. At 600 minutes (500 minutes at $200{ }^{\circ} \mathrm{C}$ ) the system appears to be highly crystalline, again showing Bragg peaks up to at least $2 \theta$ of $8^{\circ}$. Directly comparing MP AIPO-5 and HP AIPO-5 systems at both the start (Figures 3 and S7) and the end of the experiments it is hard to extract any further information, thus we now focus on the complimentary PDF data.

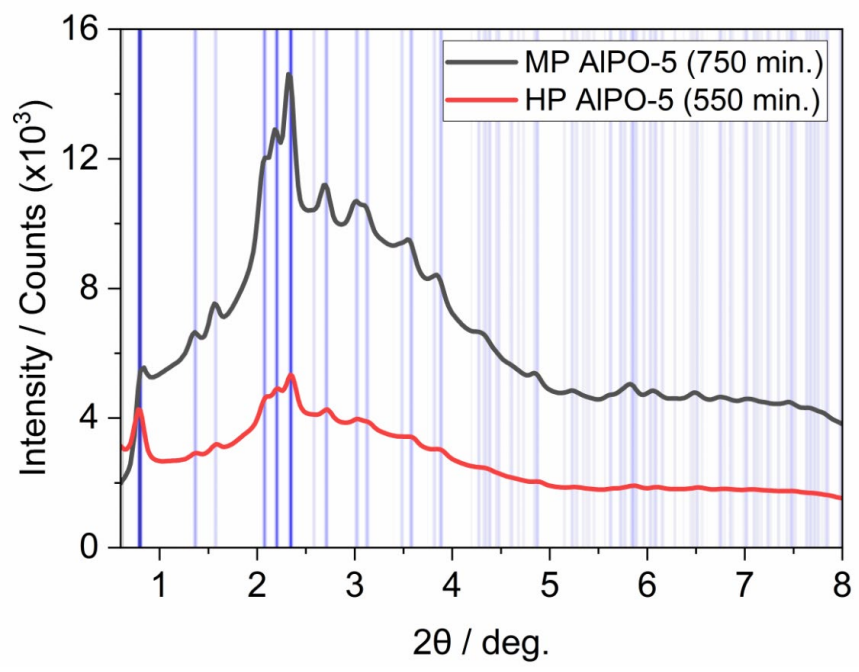

This journal is (C) The Royal Society of Chemistry 20xx
Figure 3: Direct comparisons of the stacked X-ray diffraction data for in situ MP AIPO-5 and HP AIPO-5 systems at their most crystalline. Profiles superimposed on a calculated pseudo-film pattern for crystalline AIPO-5. ${ }^{46}$

\section{Pair distribution function}

While the total scattering data can provide useful structural data, specifically on the crystallisation of ordered crystalline phases, the Fourier transform of the total scattering, the PDF, D(r), can probe the local environment of the disordered systems. This makes it incredibly well suited to focussing on the earlier pre-crystallisation regime. Here the scattering is dominated by short-range interactions such as solvation shells, intermolecular distances and emerging precrystalline structures. Initially we considered ex situ PDFs of the dry, powdered MP and HP AlPO-5 species, synthesised and calcined (template removed). This allowed us to identify what features are due to the framework itself, and which can be attributed to the reactant precursor materials or intermediate species.

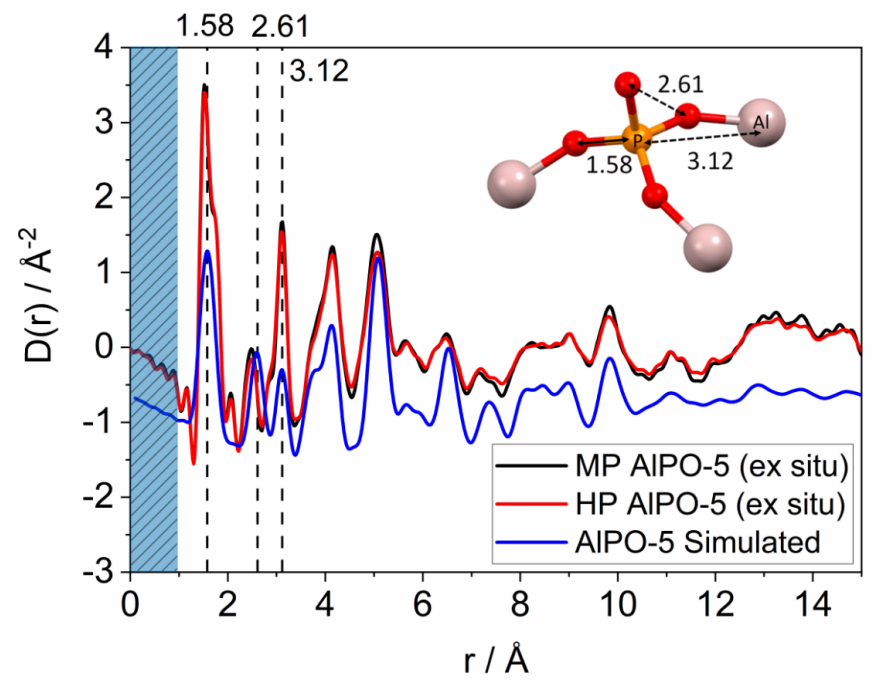

Figure 4: Comparison of the PDF data of ex situ calcined MP (black) and HP AIPO-5 (red) - showing them to be very similar, and broadly similar to the data calculated for AIPO-5 (blue). Fragment distances indicated in the inset are the median bond lengths in Å. Blue hatches indicate unreliable low-r region below $1.0 \AA$. Simulated PDF offset in $y$.

The PDF profiles (Figure 4) of the ex situ (calcined) MP and HP AIPO5 are very similar, therefore we will focus primarily on the HP AIPO5 PDF data moving forward. The PDF calculated from the single crystal structure of AIPO- $5,{ }^{46}$ is a good match to the observed bond distances in HP AIPO-5, with the exception of the low-r region which 
is the most sensitive to artefacts from counting statistics or the PDF processing. The first 3 peaks in the calculated PDF are at 1.58, 2.61 and $3.12 \AA$ respectively and likely correspond to averaged first shell $\mathrm{Al} / \mathrm{P}-\mathrm{O}$ distance (range 1.47-1.7 $\AA$ ), averaged non-bonded $\mathrm{O} \cdots \mathrm{O}$ distance (range 2.54-2.68 $\AA$ ) and averaged non-bonded Al...P distance (range 3.09-3.15). Given the significant number of possible interactions beyond this point it is challenging to make any further assignments with any great certainty.

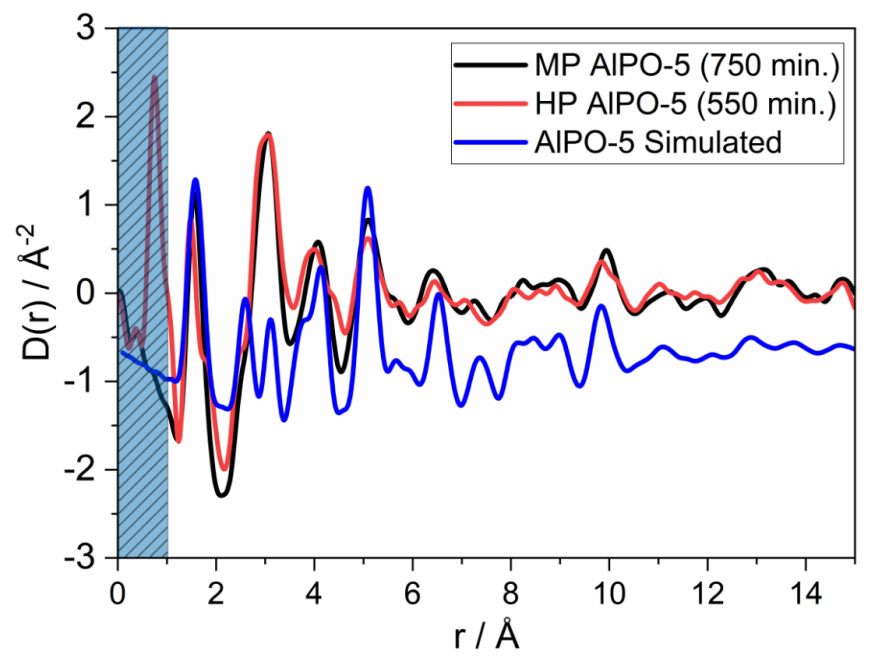

Figure 5. Comparison of the in situ MP AIPO-5(black) and HP AlPO-5 (red) PDF - showing them to be very similar, but shows significant variation in the range 2-4 $\AA$ from the calculated AIPO-5 PDF (blue). Blue hatches indicate unreliable region below $1.0 \AA$ A Simulated PDF offset.

Considering the PDF of the most crystalline in situ samples (Figure 5), we see that MP and HP AIPO-5 are in good agreement but deviate at low $r$ from the expected calculated AIPO-5 PDF. The first shell Al/P-O peak at $1.58 \AA$ is present, though now a shoulder on the high-r side of the peak is resolved, suggesting that the $\mathrm{P}-\mathrm{O}$ and $\mathrm{Al}-\mathrm{O}$ bond length distributions are becoming more discrete and separate. This could be due to the presence of water in the framework distorting the bonds, or the occupation of the pores by templates or surfactants. In contrast, the peaks at 2.61 and $3.12 \AA$ appear to have merged and shifted to slightly higher $r$, again likely due to the presence of the reagents and water. The most notable difference between the simulated and in situ data is the large feature at $2.9 \AA$. This component can be confidently attributed to the large amount of water in our hydrothermal synthesis, as confirmed from ex situ PDF data of water, measured at Southampton (Figures S8 and S9), which shows a broad feature from 2.5-3.5 A. Similarly, this same feature is present in our solution of aluminium isopropoxide, showing the aluminium does not have any long-range order in solution. However, for $r>6 \AA$ we can see that the simulated structure of the AlPO-5 dominates the PDF data, as expected, given it is the only crystalline species present. Oscillations in the $D(r)$ which are attributable to
AlPO- 5 can be seen as far out as $40 \AA$ (Figure S10), confirming the long-range order of both MP and HP AIPO-5 species.

The initial MP and HP AIPO-5 synthesis gels at room temperature (Figures S8 and S9) show excellent agreement. This is expected, as only a comparatively small quantity of DMOD is added. Contrasting the initial HP AIPO-5 gel with the initial reagents shows that the signal is dominated by water, particularly the oscillations $r>3.5 \AA$ (Figures S8 and S9). Aside from this there is little long-range order present in the system, due to the lack of crystalline material. Initially a signal is present at $\mathrm{r} \sim 1.5 \AA$. This is attributed to $\mathrm{P}-\mathrm{O}$ from the phosphoric acid (Figure S9), which is noticeably lower than the observed $1.58 \AA$ $\mathrm{Al} / \mathrm{P}-\mathrm{O}$ distance in the crystallised HP AlPO-5 system. Further we see a clearly defined signal at $1.9 \AA$ in the synthesis gel of both MP and $\mathrm{HP}$ AIPO-5, which is in agreement with a hydrated aluminium species; $\mathrm{Al}(\mathrm{OR})_{3} \cdot \mathrm{XH}_{2} \mathrm{O}$, specifically either the $\mathrm{Al}-\mathrm{O}$ or the $\mathrm{Al}-\mathrm{OH}_{2}$ bond. ${ }^{51}$

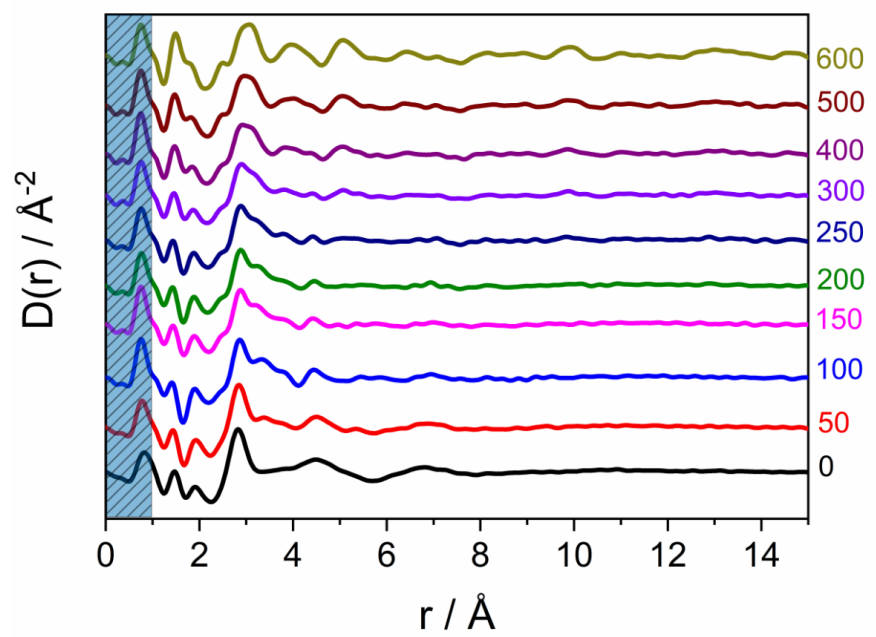

Figure 6. Stacked in situ PDF data for HP AIPO-5 during crystallisation, showing the change in the short-range PDF with crystallisation time (minutes).

The development of the PDF profile with time is most clearly observed for the HP AIPO-5 sample (Figures 6, S10 and S11), which is unaffected by the fluctuations observed in diffraction scattering intensity in the MP AIPO-5 data collections. Initial developments in the PDF after just 50 minutes can be seen, most notably the initial feature at $r=1.5 \AA$ at 0 minutes decreases and subtly shifts to a lower $r$ value (1.41 $\AA$ ) during the heating regime (0-100 minutes). This likely represents the change in $\mathrm{P}-\mathrm{O}$ bonds from $\mathrm{H}_{3} \mathrm{PO}_{4}$, to discrete $\mathrm{PO}_{4}{ }^{3-}$ units. Following this feature beyond 100 minutes shows the signal growing, and then shifting back to a higher $r$ value of $1.58 \AA$, in line with the system beginning to form $\mathrm{P}-\mathrm{O}-\mathrm{Al}$ bonds as the framework develops. Similarly, the features at $r=1.9 \AA$ at 0 minutes, assigned to hydrated aluminium species, significantly shifts to a lower $r$ value, 
and after 600 minutes coalesces with the $1.58 \AA$ feature, representing the $\mathrm{Al}-\mathrm{O}$ and $\mathrm{P}-\mathrm{O}$ bonds. Further we also see a notable shift from the water feature at $r \sim 2.9 \AA$, which after 100 minutes (just reached $200^{\circ} \mathrm{C}$ ), shows a shift to higher $r$, and appears to merge with a growing feature, starting at $4.0 \AA$ at 100 minutes. This represents the transition from PBUs $\left(\mathrm{AlO}_{4} / \mathrm{PO}_{4}\right)$ to $\mathrm{SBUs}$, as a narrower range of non-bonded $\mathrm{O} \cdots \mathrm{O}$ distances forms in the framework. As such the initial (local) framework formation seems to occur within 100-200 minutes, with the time after this dedicated to combining the SBUs to form the wider structure. Probing the longer $r$ range (Figure S11) confirms these findings, as only after 200-250 minutes can we see features beyond $15 \AA$ evolving, in good agreement with the appearance of Bragg peaks from the total scattering (Figure 2) evidencing a transition from only short-range ordering to longerrange ordering. After 250 minutes the long $r$ features continue to grow, showing that it is constantly crystallising over the whole 600 minutes.

In contrast, MP AIPO-5 appears to undergo a more rapid formation of local framework structure. Considering the time evolution of the in situ PDF data for MP AIPO-5 (Figure S12 and S13), after 100 minutes the feature at $r=1.58 \AA$ is already fully formed, with no shoulder peak visible. Similarly, the non-bonded $0 \cdots 0$ distance at $r=$ $2.9 \AA$ has already fully shifted and broadened after 100 minutes. Again, this mirrors the total scattering findings where the MP AIPO-5 framework was almost completely crystallised after just 200 minutes. This confirms that the addition of DMOD, to induce formation in HP AlPO-5 significantly slows down the framework formation, and the crystallisation kinetics. It is highly likely that hindering the crystallisation kinetics is a key feature in mesopore incorporation. If the microporous framework rapidly formed, there would be little opportunity for the mesoporogen to integrate into the framework matrix, thus would result in a strictly microporous species. By slowing down the crystallisation kinetics, the mesoporogen can interact with the growing framework, becoming part of the system. In this case, the DMOD appears to not only slow down the initial formation of the SBUs, but also the larger-scale framework growth, as witnessed by the low $r$ PDF, and high $r$ (and total scattering) respectively.

\section{Conclusions}

We have shown through a combined in situ total scattering and PDF monitoring of AIPO-5 synthesis, that the addition of the mesoporogen DMOD, significantly slows down the crystallisation kinetics over a range of length scales. We hypothesise that this is a necessary feature for a mesoporogen, in order to integrate the mesopores within the crystalline framework. It is highly likely that this notion extends beyond hierarchical systems, and into the kinetics of crystallisation for metal-doped AIPOs as well. Though PDF is currently unable to highlight the presence of the mesopores directly, the potential for more thorough analysis of the data is clear. Potential combinations with theoretical works would allow for more powerful analysis techniques, identifying the formation of specific
SBUs and distinct crystallisation regimes. Finally, we have shown, for the first time, the ability to measure the PDF in situ, during the hydrothermal synthesis of an AIPO material, with our newlydeveloped in situ hydrothermal cell.

\section{Conflicts of interest}

There are no conflicts to declare.

\section{Acknowledgements}

MEP, AEO, MEL and RR acknowledge the Total "Consortium on Metal Nanocatalysis" project for funding. SC thanks AdvanSix Inc. for their support in funding her studentship. DJMI acknowledges the EPSRC for DTP funding. In situ PDF data was collected at I15-1 at the Diamond Light Source, Didcot, UK via standard access proposals CY21878-1, EE19039-1, EE19039-2 and EE16135-1, with rapid access proposal CY22842-1.

\section{Notes and references}

1 M. Behrens, F. Studt, I. Kasatkin, S. Kühl, M. Hävecker, F. Abild-Pedersen, S. Zander, F. Girgsdies, P. Kurr, B. L. Kniep, M. Tovar, R. W. Fischer, J. K. Nørskov and R. Schlögl, Science., 2012, 336, 893-897. Y. P. Li, M. Head-Gordon and A. T. Bell, ACS Catal., 2014, 4, 1537-1545.

3 S. Bordiga, C. Lamberti, F. Bonino, A. Travert and F. Thibault-Starzyk, Chem. Soc. Rev., 2015, 44, 7262-7341.

N. Pienack and W. Bensch, Angew. Chemie - Int. Ed., 2011 50, 2014-2034.

M. G. O'Brien, A. M. Beale and B. M. Weckhuysen, Chem. Soc. Rev., 2010, 39, 4767-4782.

J. Dou, Z. Sun, A. A. Opalade, N. Wang, W. Fu and F. Tao, Chem. Soc. Rev., 2017, 46, 2001-2027.

G. Férey, M. Haouas, T. Loiseau and F. Taulelle, Chem. Mater., 2014, 26, 299-309.

L. Gómez-Hortigüela, F. Corà, C. R. A. Catlow and J. PérezPariente, J. Am. Chem. Soc., 2004, 126, 12097-12102.

A. Aerts, C. E. A. Kirschhock and J. A. Martens, Chem. Soc. Rev., 2010, 39, 4626-4642.

M. Moliner, F. Rey and A. Corma, Angew. Chemie - Int. Ed., 2013, 52, 13880-13889.

M. Zaarour, B. Dong, I. Naydenova, R. Retoux and S. Mintova, Microporous Mesoporous Mater., 2014, 189, 1121.
L. Tosheva and V. P. Valtchev, Chem. Mater., 2005, 17, 
2494-2513.

13 S. K. Gupta, A. C. Kalita, A. A. Dar, S. Sen, G. N. Patwari and R. Murugavel, J. Am. Chem. Soc., 2017, 139, 59-62.

M. E. Potter, J. Kezina, R. Bounds, M. Carravetta, T. M. Mezza and R. Raja, Catal. Sci. Technol., 2018, 8, 51555164.

C. S. Cundy and P. A. Cox, Microporous Mesoporous Mater., $2005,82,1-78$

J. Z. Hu, M. Y. Hu, Z. Zhao, S. Xu, A. Vjunov, H. Shi, D. M. Camaioni, C. H. F. Peden and J. A. Lercher, Chem. Commun., 2015, 51, 13458-13461. C, 2016, 120, 1701-1708.

A. Chawla, N. Linares, J. D. Rimer and J. García-Martínez, Chem. Mater., 2019, 31, 5005-5013.

R. Brent, P. Cubillas, S. M. Stevens, K. E. Jelfs, A. Umemura, J. T. Gebbie, B. Slater, O. Terasaki, M. A. Holden and M. W. Anderson, J. Am. Chem. Soc., 2010, 132, 13858-13868.

K. Simmance, G. Sankar, R. G. Bell, C. Prestipino and W. Van Beek, Phys. Chem. Chem. Phys., 2010, 12, 559-562.

$\varnothing$. B. Vistad, D. E. Akporiaye and K. P. Lillerud, J. Phys. Chem. B, 2001, 105, 12437-12447.

P. P. E. A. De Moor, T. P. M. Beelen and R. A. Van Santen, J. Phys. Chem. B, 1999, 103, 1639-1650.

M. E. Potter, J. M. Purkis, M. Perdjon, P. P. Wells and R. Raja, Mol. Syst. Des. Eng., 2016, 1, 335-344.

A. M. Beale, M. G. O’Brien, M. Kasunič, A. Golobič, M. Sanchez-Sanchez, A. J. W. Lobo, D. W. Lewis, D. S. Wragg, S. 42 Nikitenko, W. Bras and B. M. Weckhuysen, J. Phys. Chem. C, 2011, 115, 6331-6340.

A. M. Beale, A. M. J. Van Der Eerden, D. Grandjean, A. V. Petukhov, A. D. Smith and B. M. Weckhuysen, Chem. Commun., 2006, 4410-4412.

J. M. Thomas, Angew. Chemie - Int. Ed., 1999, 38, 35883628.

P. Norby, A. Nørlund Christensen and J. C. Hanson, Inorg. Chem., 1999, 38, 1216-1221.

G. Agostini, C. Lamberti, L. Palin, M. Milanesio, N. Danilina, B. Xu, M. Janousch and J. A. Van Bokhoven, J. Am. Chem. Soc., 2010, 132, 667-678.

D. Grandjean, A. M. Beale, A. V. Petukhov and B. M. Weckhuysen, J. Am. Chem. Soc., 2005, 127, 14454-14465.

D. A. Keen, J. Appl. Crystallogr., 2001, 34, 172-177.

T. Egami and S. Billinge, Underneath the Bragg Peaks, Elsevier, Amsterdam, 2nd Edition, 2012.
P. Juhás, T. Davis, C. L. Farrow and S. J. L. Billinge, J. Appl. Crystallogr., 2013, 46, 560-566.

M. M. Martínez-Iñesta, I. Peral, T. Proffen and R. F. Lobo, Microporous Mesoporous Mater., 2005, 77, 55-66.

W. J. Roth, P. Nachtigall, R. E. Morris, P. S. Wheatley, V. R. Seymour, S. E. Ashbrook, P. Chlubná, L. Grajciar, M. Položij, A. Zukal, O. Shvets and J. Čejka, Nat. Chem., 2013, 5, 628633.

D. S. Firth, S. A. Morris, P. S. Wheatley, S. E. Russell, A. M. Z. Slawin, D. M. Dawson, A. Mayoral, M. Opanasenko, M. Položij, J. Čejka, P. Nachtigall and R. E. Morris, Chem. Mater., 2017, 29, 5605-5611.

S. E. Henkelis, S. A. Morris, M. Mazur, P. S. Wheatley, L. N. McHugh and R. E. Morris, J. Mater. Chem. A, 2018, 6, 17011-17018.

H. Yamada, S. Tominaka, K. Ohara, Z. Liu, T. Okubo and T. Wakihara, J. Phys. Chem. C, 2019, 123, 28419-28426.

K. Na, C. Jo, J. Kim, K. Cho, J. Jung, Y. Seo, R. J. Messinger, B. F. Chmelka and R. Ryoo, Science (80-. )., 2011, 333, 328332.

C. E. A. Kirschhock, S. P. B. Kremer, J. Vermant, G. Van Tendeloo, P. A. Jacobs and J. A. Martens, Chem. - A Eur. J., 2005, 11, 4306-4313.

L. H. Chen, X. Y. Li, J. C. Rooke, Y. H. Zhang, X. Y. Yang, Y. Tang, F. S. Xiao and B. L. Su, J. Mater. Chem., 2012, 22, 17381-17403.

S. Tao, X. Li, H. Gong, Q. Jiang, W. Yu, H. Ma, R. Xu and Z. Tian, Microporous Mesoporous Mater., 2018, 262, 182190.

S. H. Newland, W. Sinkler, T. Mezza, S. R. Bare, M. Carravetta, I. M. Haies, A. Levy, S. Keenan and R. Raja, ACS Catal., 2015, 5, 6587-6593.

I. Miletto, G. Paul, S. Chapman, G. Gatti, L. Marchese, R. Raja and E. Gianotti, Chem. - A Eur. J., 2017, 23, 99529961.

J. Filik, A. W. Ashton, P. C. Y. Chang, P. A. Chater, S. J. Day, M. Drakopoulos, M. W. Gerring, M. L. Hart, O. V. Magdysyuk, S. Michalik, A. Smith, C. C. Tang, N. J. Terrill, M. T. Wharmby and H. Wilhelm, J. Appl. Cryst., 2017, 50, 959966

A. K. Soper and E. R. Barney, J. Appl. Crystallogr., 2011, 44, 714-726.

S. Qiu, W. Pang, H. Kessler and J. L. Guth, Zeolites, 1989, 9, 440-444.

P. A. Barrett, G. Sankar, C. R. A. Catlow and J. M. Thomas, J. Phys. Chem. Solids, 1995, 56, 1395-1495.

J. Bauer, T. Selvam, J. Ofili, E. Che, R. Herrmann and W. 
Journal Name

Schwieger, Stud. Surf. Sci. Catal., 2007, 170, 837-844.

49 H. K. D. Nguyen, T. N. Don, G. Sankar and R. A. Catlow, Catal. Commun., 2012, 25, 125-129.

50

T. Ikeda, K. Miyazawa, F. Izumi, Q. Huang and A. Santoro, J.

Phys. Chem. Solids., 1999, 60, 1531-1535.

51 N. H. de Leeuw and S. C. Parker, J. Am. Ceram. Soc., 1999,

82, 3209-3216. 